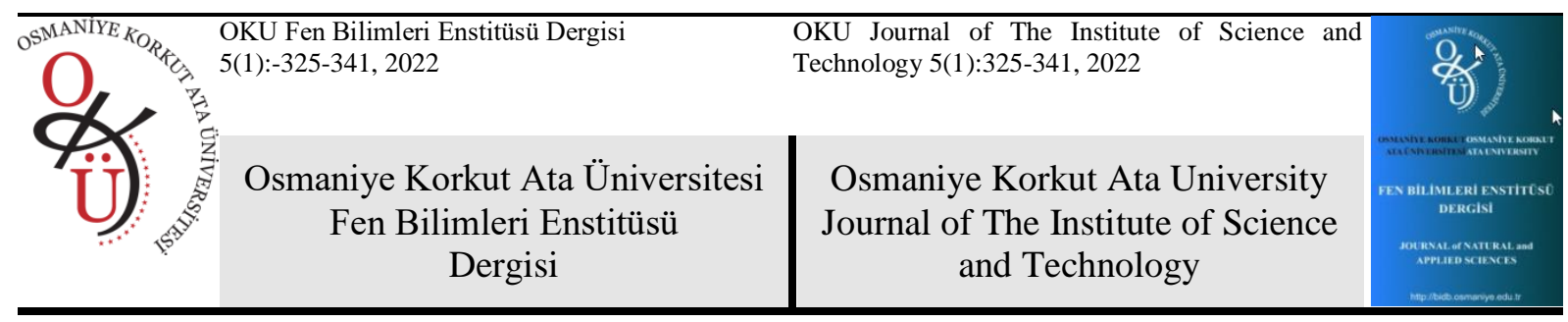

Algorithmic Workspace Programming of the Collaborative Multi-Robots

\author{
Haydar ŞAHIIN ${ }^{1 *}$ \\ ${ }^{1}$ İstanbul Gedik Üniversitesi Mühendislik Fakültesi Mekatronik Mühendisliği Bölümü, İstanbul \\ ${ }^{1}$ https://orcid.org/0000-0001-8435-9448 \\ *Corresponding author: haydar.sahin@gedik.edu.tr
}

\section{Research Article}

Article History:

Received: 30.11 .2021

Accepted: 05.02.2022

Published online:08.03.2022

\section{Keywords:}

Workspace

Collaborative robots

Modular mechanism

Multi-robots

\begin{abstract}
In the present study, the Controllable Instantaneous Screw Axes (C-ISA) 1 and C-ISA 2 shape variable angles are modified independently to realize various rule-based work spaces and trajectories for multi collaborative robot control. The toolbox developed previously is used to obtain the algorithm of the workspaces for 2-RR collaborative multi-robots herein. Six collaborative multi-robots are exploited to design the intersecting workspaces with generated trajectories. The classifications of the workspaces are unveiling the boundaries of the collaborations for the six multi-robots of the 2-RR (Revolute Revolute). The recent developments are showing that the multi-robots are embedding into the automation systems to achieve the novel requirements of the challenging technology. Therefore, the workspace algorithms developed herein are prepared to be utilized by these automation systems.
\end{abstract}

\title{
İşbirlikçi Çoklu Robotların Algoritmik Çalışma Alanı Programlaması
}

\section{Araştırma Makalesi}

Makale Tarihçesi:

Gelis tarihi: 30.11 .2021

Kabul tarihi: 05.02.2022

Online Yayınlanma:08.03.2022

Anahtar kelimeler

Çalışma alanı

İsbirlikçi robotlar

Modüler mekanizma

Çoklu robotlar

\section{ÖZ}

Bu çalışmada, Kontrol Edilebilir Anlık Vida Eksenleri (C-ISA) 1 ve C-ISA 2 şekil değişken açıları, çoklu işbirlikçi robot kontrolü için çeşitli kural tabanlı çalışma alanları ve yörüngeleri gerçekleştirmek için bağımsız olarak değiştirilir. Daha önce geliştirilen araç kutusu, buradaki 2-RR işbirlikçi çoklu robotlar için çalışma alanlarının algoritmasını elde etmek için kullanılmaktadır. Oluşturulan yörüngelerle kesişen çalışma alanlarını tasarlamak için altı işbirlikçi çoklu robottan yararlanılır. Çalışma alanlarının sınıflandırmaları, 2-RR'nin (Revolute Revolute) altı çoklu robotu için işbirliklerinin sınırlarını ortaya koyuyor. Son gelişmeler, zorlu teknolojinin yeni gereksinimlerini karşılamak için çoklu robotların otomasyon sistemlerine yerleştirildiğini gösteriyor. $\mathrm{Bu}$ nedenle burada geliştirilen çalışma alanı algoritmaları bu otomasyon sistemlerinde kullanılmak üzere hazırlanmıştır.

To Cite: Sahin H.. Algorithmic Workspace Programming of the Collaborative Multi-Robots. Osmaniye Korkut Ata

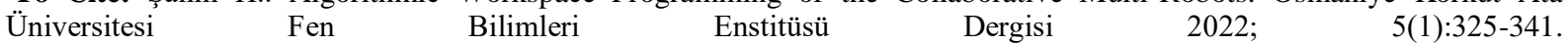

\section{Introduction}

Programmable workspaces are required for collaborative robots. These algorithms are for industrial production, automated factories, industrialization (Clark and Rojas, 2020), automation (Queralta et al., 2020), construction (Rajesh and Anandu, 2020), search and rescue robots (Krizmancic et al., 2020). Multi-purpose robots with varied stiffness capabilities are requested for task space abilities (Clark and Rojas, 2020). The production line includes many robots operating synchronously (concurrently) to assemble, pick and place the parts of the products. The programmability for multi-purpose products is 
required for industrialization (Clark and Rojas, 2020). Serial robots with the advantage of the synchronization of the multi-robots are widespread in the production lines.

The created workspaces are classified to be used in the modular collaborative robot design (Sahin, 2021). The collaborative robot of skew revolute joints has high reachability for defined task spaces (Elbanhawi and Simic, 2020; Feng et al., 2020; Olesen et al., 2020; Su et al., 2020). Additionally, the soft robotic link integrates to increase the reachability of the collaborative robots (Clark and Rojas, 2020). However, rigid adjustable or controllable joints can be an invaluable design option for collaborative robots. The programmability is provided with the changeable variables of the $\alpha_{1-4}$ mechanism for the collaborative robots.

The goal herein is to include the least number of revolute joints for a compact design to obtain the required precise workspaces or trajectories for the collaborative multi-robots. The higher quantity of the joints causes a higher risk of accidents for the multi-collaborative robots. Upon exploiting the skew revolute joints, the 2-RR multi-collaborative robots contain highly maneuverable joints. Additionally, having designed the modular multi-collaborative robots with 2-RR spatial mechanisms, the combination of the modular workspaces leads to generating the modular spatial chain.

Therefore, the 2-RR macro modular collaborative multi-robots are chosen as fundamental serial mechanisms which contain the minimum number of joints for a spatial robotic. Thus, the skew revolute joints can reach versatile workspaces compared to the non-skew revolute joints with the least quantity of the joints. However, the adjustability of the joint for skewness is required to arrange the varied skew angles of the revolute joint. The rule-based workspace design covered in this study is related to the relative rule definitions between the C-ISA angles of the shape variables of the $\alpha_{1-4}$ and $\theta_{1,2}$ for the C-manifold skew revolute mechanism of the collaborative robots. The parametric rules define the relation between the angles of the shape variables for the collaboration of the robot mechanisms.

The algorithms specialize in manipulating the multi-robots for path following, path planning, and sensory data gathering (Rajesh and Anandu, 2020). The trajectories define the distance measurement techniques on the paths (Su et al., 2020). Collaborative robot interaction with grippers is the main subject covered as decision-making for automation systems in the article (Su et al., 2020).

The automation system of the Industry 4.0 shall contain the repository of the obstacle space (Olesen et al., 2020), Configuration space (C-space) (Olesen et al., 2020), and the multi-robot configuration spaces as described in this article. Having constructed the conceptual animation-based workspaces, they are aiding to visualize the robot motion planning. Additionally, they are confining the algorithmic programming process of the multi-robots for collaborative robots. Collaborative multi-robots are specified to work coordinately with fixed mechanisms and mobile robots (Elbanhawi and Simic, 2014). The research in this field states the importance of the coordinated functioning of the multi collaborative robots (Feng et al., 2020). Thus, the workspace of the multi-collaborative robots is 
emerging to determine the overlapping and non-overlapping regions for alternative algorithms covered in this research article.

The learning algorithms are developed for cleaning and maintenance robots (Yang et al., 2020). The algorithms of the path planning are specified based on the design requirements via functions (Lakshmanan et al., 2020). The soft robot gripper composes the design of the power grasp (Dilibal et al., 2021). Upon determining the speed and separation monitorings for the safe speeds,

the workspaces of the collaborative robots investigate with conditional results in the ISO/TS15066 standard (Byner et al., 2019; Le et al., 2020; Wang and Meng, 2020). Collision avoidance (Marvel and Norcross, 2017), trajectory generation (ISO/TS15066:2016, 2016), and deep-learning (Moe et al., 2020) apply for the inverse and forward kinematics of the robots. Kinematic (Henderson and Prazenica, 2021) and inverse kinematics (Wang et al., 2021) formulas are methods of developing the programming of multi-collaborative robots. The robot-based programmable (Thalamy et al., 2019; Martínez and Campa, 2021; Xiao et al., 2021) systems determine the reconfiguration methods. The purpose of this study is generation of the collaborative multi-robot workspaces systematically using the developed mathematical algorithms of the Lie algebra.

\section{Methodology}

Recently, the SMILAT (Symbolic Mathematics with Integrated Lie Algebra Toolbox) was developed as a Lie Algebraic Formulation of the Kinematics and Dynamics for Robots as a Toolbox (LAFKIDROT) as described in the reference of (Sahin, 2021). This toolbox is used to develop the algorithm of the workspaces for 2-RR collaborative multi-robots herein. The surface plot is utilized for workspace determination via LAFKID-ROT. The intersection of the planned path trajectory is observed with the created workspaces of the animations. The modular design approach is utilized for the LAFKID-ROT.

\section{The Modular C-Manifold Design of Workspaces with the C-ISA Angles for 2-RR Spatial Mechanisms}

The workspace analyses of configuration-dependent multiple synchronous C-ISA are obtained for group angular parameters of the 2-RR modular robots avoiding the collision using nonoverlapping workspaces in the result section. The $\alpha_{1-4}$ can be arranged for the designed group of the 2-RR robots so that reconfiguration workspaces define the required multiple workspaces. The workspaces are definable via Maxima, Octave, and Matlab. The workspaces with overlapping or without overlapping are created applying rule-based mathematical ratios among the $\alpha_{1-4}$ angles. The $5 \pi / 6$ radian for the $\theta_{1}$ and $\theta_{2}$ arranged considering the dead region for the workspace of the 2-RR modular robot designs. The $x y, y z, x z$, and $x y z$ surfaces of the workspaces are plotted in Figure 3a using the algorithm of the $\alpha_{1,2}=\mathrm{i} . \pi / 3, \alpha_{3,4}=\mathrm{i} . \pi / 6$ for $\mathrm{i}=1-6$ for the generalized Equation of $A d_{\left(g_{\left.1 j_{2}\right)}\right)} \xi_{1 j_{-} 2 j}^{S}$. 


\section{Programmable workspace analyses of the collaborative robots for macro modular C-manifold 2-}

\section{RR spatial mechanisms}

The previous study mainly focused on the defined constant angle between the C-ISA twists [5]. The article herein does not contain the constant angle between the C-ISA 1 and 2. Thus, the C-ISA 1 and C-ISA 2 shape variable angles are modified independently to realize various rule-based workspaces and trajectories for multi collaborative robot control. The skew twist angles of the joints $\alpha_{1}, \alpha_{2}$ for the first joint and $\alpha_{3}, \alpha_{4}$ for the second joint in Figure 1. These parametric angles are used to describe the workspaces in the following sections.

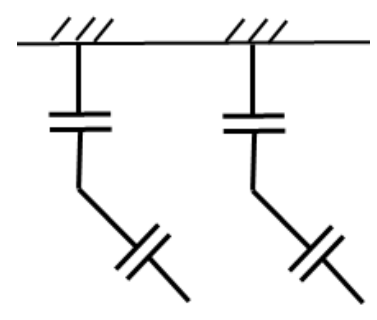

Figure 1. The robots with $\alpha_{1}, \alpha_{2}$ and $\alpha_{3}, \alpha_{4}$ angles for the joints of two robots: $2 \|$ hh - 2 ॥

The serial collaborative multi-robot task spaces are for the design and programming of the workspaces for the modular design of 2-RR serial robot systems. The end effector joint and spatial second joint twists with adjoints define the $\alpha_{1}, \alpha_{2}, \alpha_{3}$, and $\alpha_{4}$ for workspace analyses of ( $\left.\operatorname{SE}(3) \times S^{1} \mathrm{xS}^{1}\right) \mathrm{C}$-manifold using Lie algebra based toolbox (Sahin, 2021).

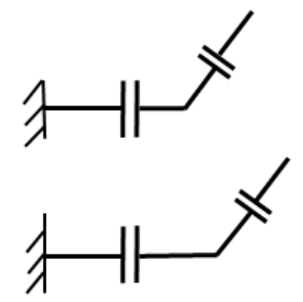

a) 4-RRRR: 2 || vv - 2 ॥
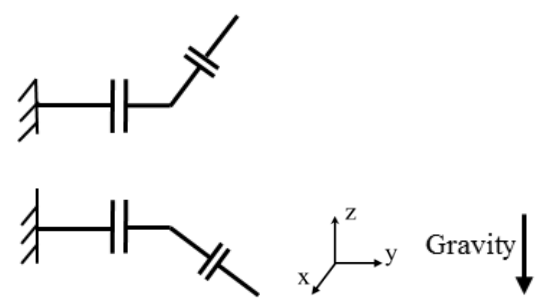

b) 4-RRRR: 2 || vv - $2 \perp$

Figure 2. The configurations of the 4-RRRR parallel robots generated by the integration of the two of the modular 2-RR collaborative multi-robots.

The defined values are $\theta_{1}$ and $\theta_{2}=5 \pi / 6$ radian with $i=1-6$ for the workspaces described by $A d_{\left(g_{1 j_{2} 2}\right)} \xi_{1 j_{2} 2 j}^{S}$. The workspaces of C-manifolds are for the C-ISA 1 of $\alpha_{1,2}$ and for the C-ISA 2 of $\alpha_{3,4} \cdot$ 
Table 1. Specifications for the plots of both the variable order and value based algorithm

\begin{tabular}{llllllll}
\hline Algorithm & $\alpha_{1}$ & $\alpha_{2}$ & $\alpha_{3}$ & $\alpha_{4}$ & $\theta_{1}$ & $\theta_{2}$ & $\mathrm{i}$ \\
\hline $\mathrm{a}$ & $i . \pi / 3$ & $i . \pi / 3$ & $i . \pi / 6$ & $i . \pi / 6$ & $5 \pi / 6$ & $5 \pi / 6$ & $1-6$ \\
$\mathrm{~b}$ & 0 & 0 & $\mathrm{i} . \pi / 100$ & $\mathrm{i} . \pi / 100$ & $5 \pi / 6$ & $5 \pi / 6$ & $1-6$ \\
$\mathrm{c}$ & $\mathrm{i} . \pi /(10)$ & $\mathrm{i} . \pi /(10)$ & $\mathrm{i}^{*} \pi / 100$ & $\mathrm{i}^{*} \pi / 100$ & $5 \pi / 6$ & $5 \pi / 6$ & $1-6$ \\
$\mathrm{~d}$ & 0 & 0 & $\mathrm{i} . \pi /(10)$ & $\mathrm{i} . \pi /(10)$ & $5 \pi / 6$ & $5 \pi / 6$ & $1-6$ \\
$\mathrm{e}$ & 0 & 0 & $\mathrm{i} . \pi / 10$ & $10 \pi /(\mathrm{i}+1)$ & $5 \pi / 6$ & $5 \pi / 6$ & $1-6$ \\
$\mathbf{f}$ & $\mathrm{i} . \pi / 100$ & 0 & $\mathrm{i} . \pi / 100$ & 0 & $5 \pi / 6$ & $5 \pi / 6$ & $1-6$ \\
$\mathbf{g}$ & 0 & $\mathrm{i} . \pi / 10$ & 0 & $\mathrm{i} . \pi / 10$ & $5 \pi / 6$ & $5 \pi / 6$ & $1-6$ \\
$\mathbf{h}$ & $\mathrm{i} . \pi / 30$ & $\mathrm{i} . \pi / 30$ & $\mathrm{i} . \pi / 20$ & $\mathrm{i} . \pi / 20$ & $5 \pi / 6$ & $5 \pi / 6$ & $1-6$ \\
$\mathbf{i}$ & 0 & $\mathrm{i} . \pi / 10$ & 0 & $\mathrm{i} . \pi / 10$ & $5 \pi / 6$ & $5 \pi / 6$ & $1-6$ \\
$\mathbf{k}$ & $\mathrm{i} . \pi / 10$ & $\mathrm{i} . \pi / 10$ & $\mathrm{i} . \pi / 10$ & $\mathrm{i} . \pi / 10$ & $5 \pi / 6$ & $5 \pi / 6$ & $1-6$ \\
\hline
\end{tabular}

The parameters are $\left(\theta_{1}, \theta_{2}, \alpha_{1}, \alpha_{2}, \alpha_{3}, \alpha_{4}\right), i=1-6$, for the equations in Table 1 . The $\theta_{1}$ and $\theta_{2}=5 \pi / 6$ are radian. The algorithm of the design process is described step by step with algorithm 1 of Figure 3 for the generation of the spatial workspace of the multi-collaborative 2-RR robots.

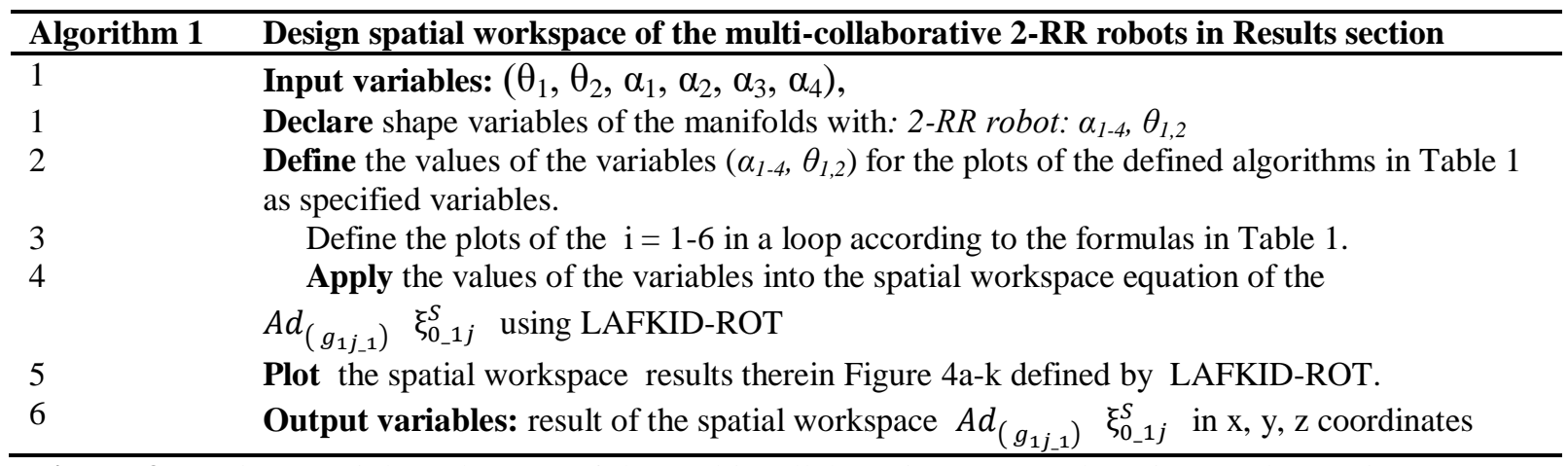

Figure 3. Design spatial workspace of the multi-collaborative 2-RR robots in Results section

Algorithm 1 indicates the steps of the workspace design, as in Figure 4.

\section{The Results of Workspaces with the independent C-ISA Angles for Collaborative Multi-Robot} Programming of 2-RR

In this subsection, I will characterize the $\theta_{1,2}$ and $\alpha_{1-4}$ for macro modular C-manifold. These parameters are six configurations that designate the regular motion patterns for an algorithm of task spaces in robot kinematics, as shown in Figures 1 and 2. The ranges of the $\theta_{1}$ and $\theta_{2}$ are $0-5 \pi / 6$ radian again. The spatial shape variables define the parameters of the $\alpha_{1-4}$ while the reduced shape variables are selected as $\theta_{1}$ and $\theta_{2}$. The workspaces for the 2-RR modular robots configure the results using the kinematic equation of the $A d_{\left(g_{\left.1 j_{2}\right)}\right)} \xi_{1 j_{2} 2 j}^{S}$ as in the following sections.

Previously, the constant constraint angle in between the C-ISA twists was utilized for the workspace design (Sahin, 2021). The study herein is aiming the algorithmic workspace programming without 
considering the constant angle between C-ISA 1 and 2. The rules herein are arranged with the defined six workspace regions of the algorithmic formula dependent on the ratio of $i$ as shown in Table 1 . These scenarios are an indication of the rules of the overlapping conditions algorithmically which are applicable to the collaborative multi-robots.

Depending on the design of the 2-RR robots, overlapping might require specific trajectory path planning of the macro modular structures for the interaction of the multiple robots.

Similarly, the workspaces without overlapping for six 2-RR modular robots are required for collision avoidance purposes in path planning. The overlapping causes the collaborative work of multiple robots. The macro modular $C$-manifolds of 2-RR modular robots can select a configuration-dependent workspace from the classified group of Figure 3. Upon defining the workspace overlapping between each pair of robots, the six modular robots are designed accordingly.

The rule-based equations for the C-ISA 1 and 2 angles are defined as in Figure 3 to create the reachable workspaces with specified conditions as in the Table 1. These conditions, as shown in Table 1 for Figure 3, can be classified as

1. non-overlapping of any macro modular workspaces (a-e),

2. overlapping on the edge of the workspaces $(\mathbf{f}-\mathbf{g})$, and

3. overlapping in the middle of the workspaces $(\mathbf{h}-\mathbf{k})$.

Upon overlapping the workspaces utilized for transitions from one workspace to another, workspaces are in path planning of the macro modular structures of the 2-RR modular robots for the equations in Table 1.

The rule-based constraint equations are defined with the relative angular position of the $\alpha_{1-4}$. This rule-based relativity is based on the relative angular positions of the $\alpha_{1}, \alpha_{2}, \alpha_{3}$, and $\alpha_{4}$ for the macro modular $C$-manifolds. The rule-based constraints of the C-ISA 1 and 2 are in the Figures of the (3a-3h) as the $\left(\alpha_{1,2}-\alpha_{3,4}\right),\left(\alpha_{1,3}-\alpha_{2,4}\right)$ and $\left(\alpha_{1,2}-\alpha_{3}-\alpha_{4}\right)$. The mathematical rules are designed by the relative positions between $\alpha_{1,2}$ and $\alpha_{3,4}$, between $\alpha_{1,3}$ and $\alpha_{2,4}$. Similarly, the relative positions are arranged among $\alpha_{1,2}, \alpha_{3}$, and $\alpha_{4}$ shape variables. The specified characteristics of the overlappings are explained below.

1. The regional overlapping on the common diagonal axis in Figure $3 \mathbf{h}$,

2. The regional overlapping on the common axis parallel to y in Figure $3 \mathbf{i}$,

3. Multiple regional overlappings in Figure $3 \mathbf{k}$,

4. Overlapping on the one edge of $y$ in Figure $3 \mathbf{g}$,

5. Overlappings on the two edges of $\mathrm{z}$ in Figure $3 \mathbf{f}$.

Rules of the C-ISA 1 and 2 are for C-manifolds as in the Equation of the $A d_{\left(g_{1 j_{-} 1}\right)} \xi_{0_{-} 1 j}^{S}$

The $\left(A d_{\left(g_{\left.1 j_{-}\right)}\right)} \xi_{1 j_{-} 2 j}^{S}\right)$ of workspaces is for six shape variables $\left(\alpha_{1-4} \theta_{1,2}\right)$ of the two reduced shape variables $\theta_{1}$ and $\theta_{2}$. The geometric parameters are $l_{1}=0.2$ meter and $r_{1}=r_{2}=0.1$ meter for the Figure 3 . The task spaces are identified with the shape variables $\alpha_{1-4}$ and $\theta_{1,2}$. The reduced shape variables 
of $\theta_{1,2}$, with $5 \pi / 6$ radians, are used to rotate around the skew axes created via $\alpha_{1-4}$ for macro modular C-manifolds of 2-RR robots. The collar code is as follows.

Dark Blue: 1, Brown: 2, Red: 3, Yellow: 4, Light Blue: 5, Green: 6

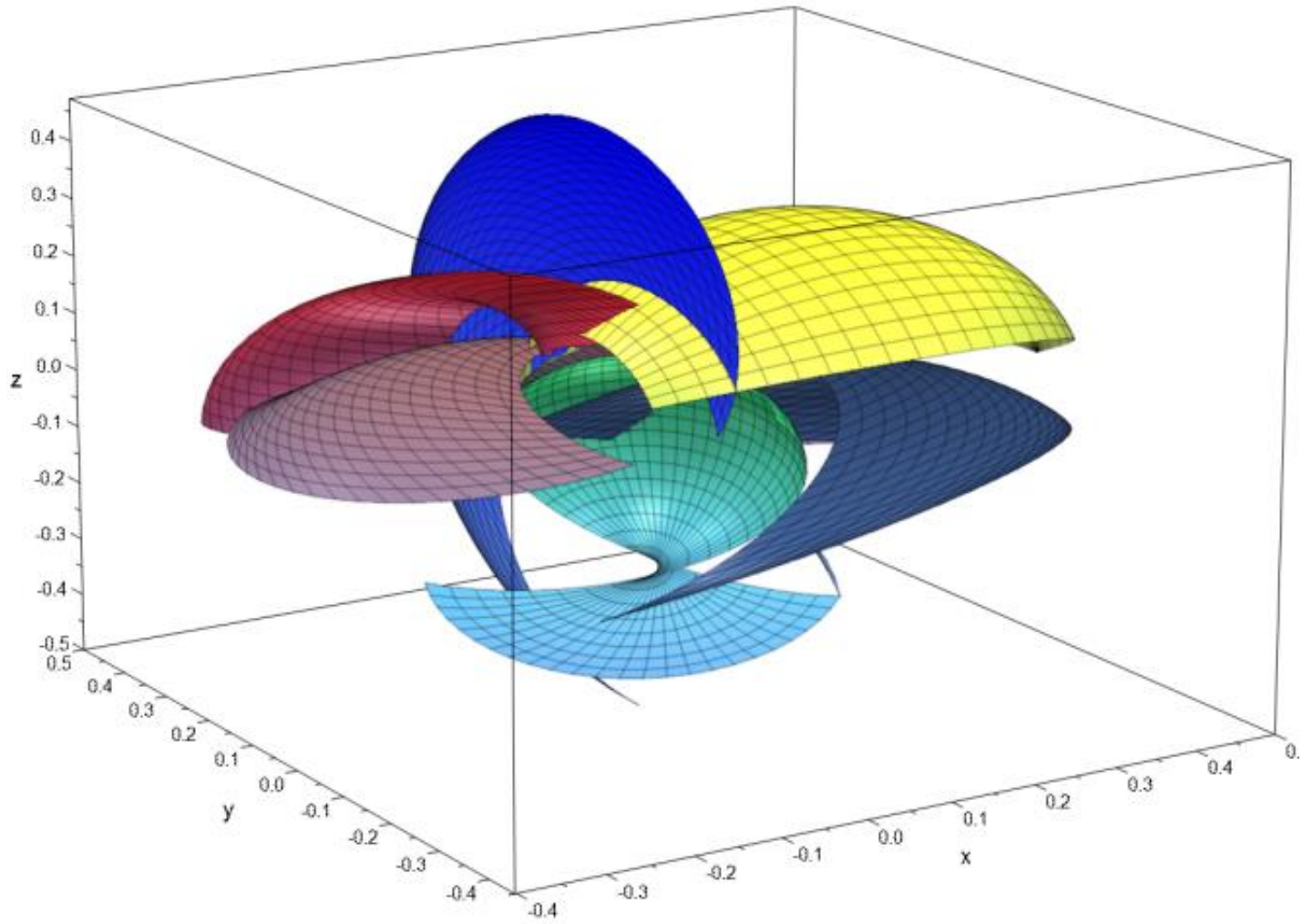

4a) The workspaces of C-manifolds for C-ISA 1 of $\alpha_{1,2}=i . \pi / 3$ and C-ISA 2 of $\alpha_{3,4}=i . \pi / 6$. 
Dark Blue: 1, Brown: 2, Red: 3, Yellow: 4, Light Blue: 5, Green: 6

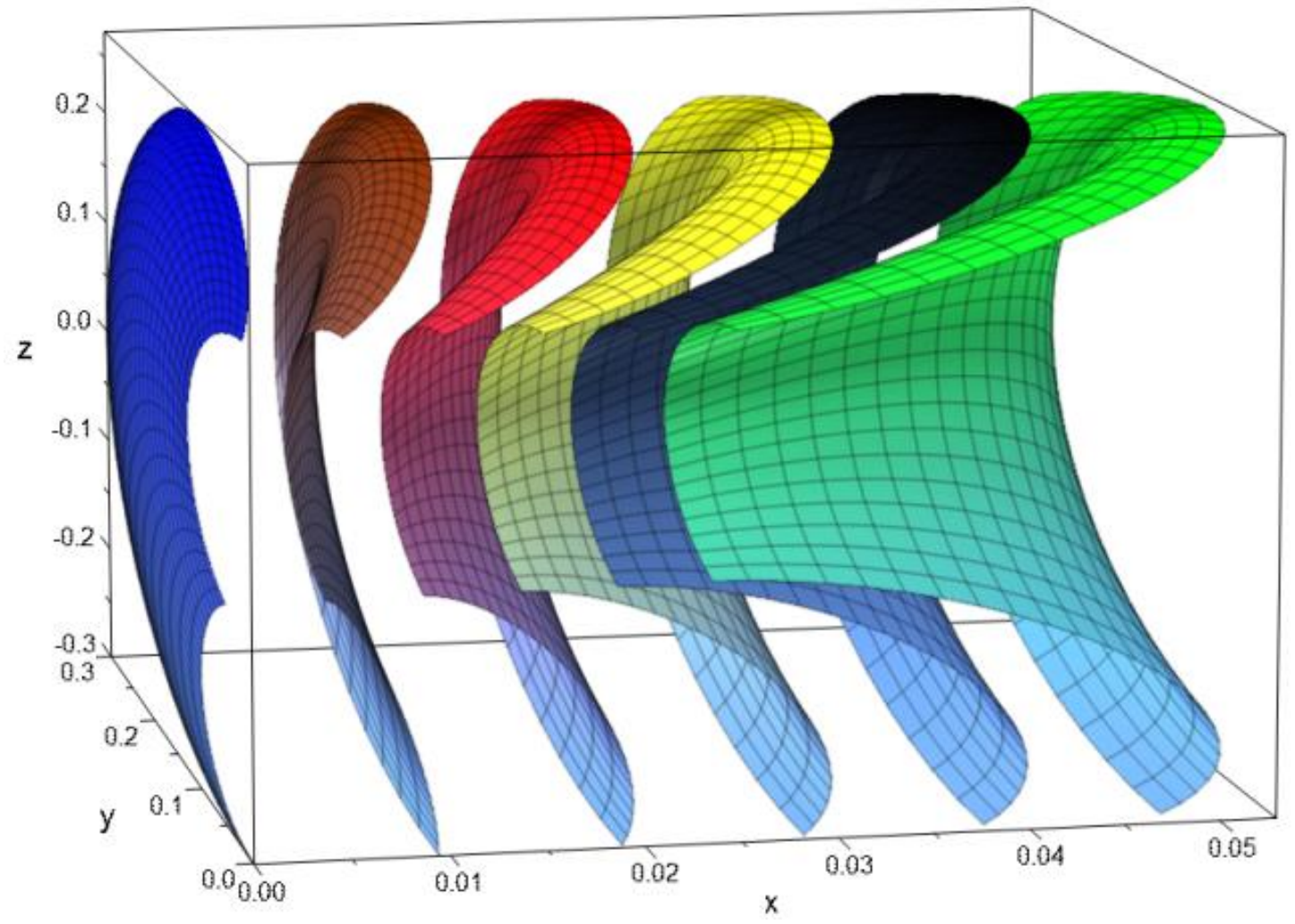

4b) The workspaces of C-manifolds for C-ISA 1 of $\alpha_{1,2}=0$ and C-ISA 2 of $\alpha_{3,4}=\mathrm{i} . \pi / 100$.

Dark Blue: 1, Brown: 2, Red: 3, Yellow: 4, Light Blue: 5, Green: 6

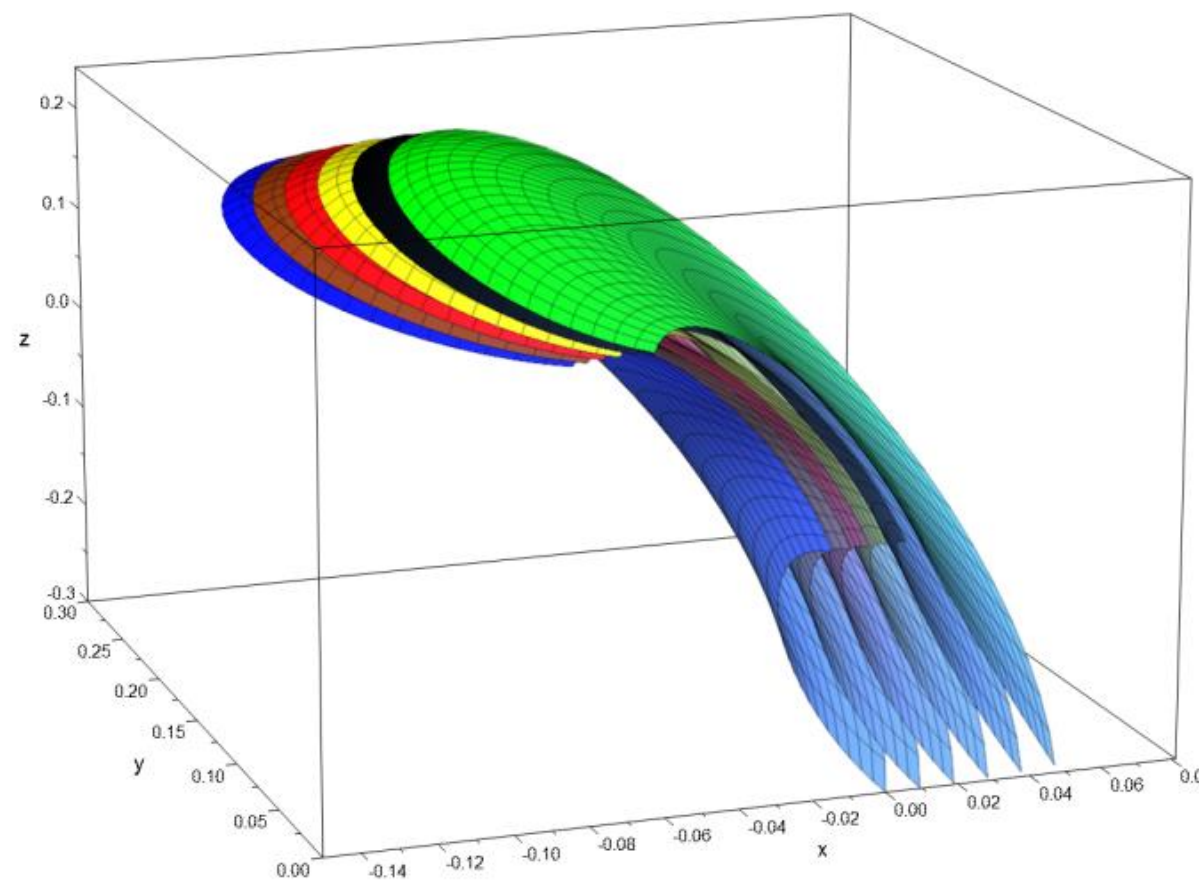

4 c) The workspaces of C-manifolds for C-ISA 1 of $\alpha_{1,2}=\mathrm{i} . \pi /(10)$, C-ISA 2 of $\alpha_{3,4}=i^{*} \pi / 100$. 
Dark Blue: 1, Brown: 2, Red: 3, Yellow: 4, Light Blue: 5, Green: 6

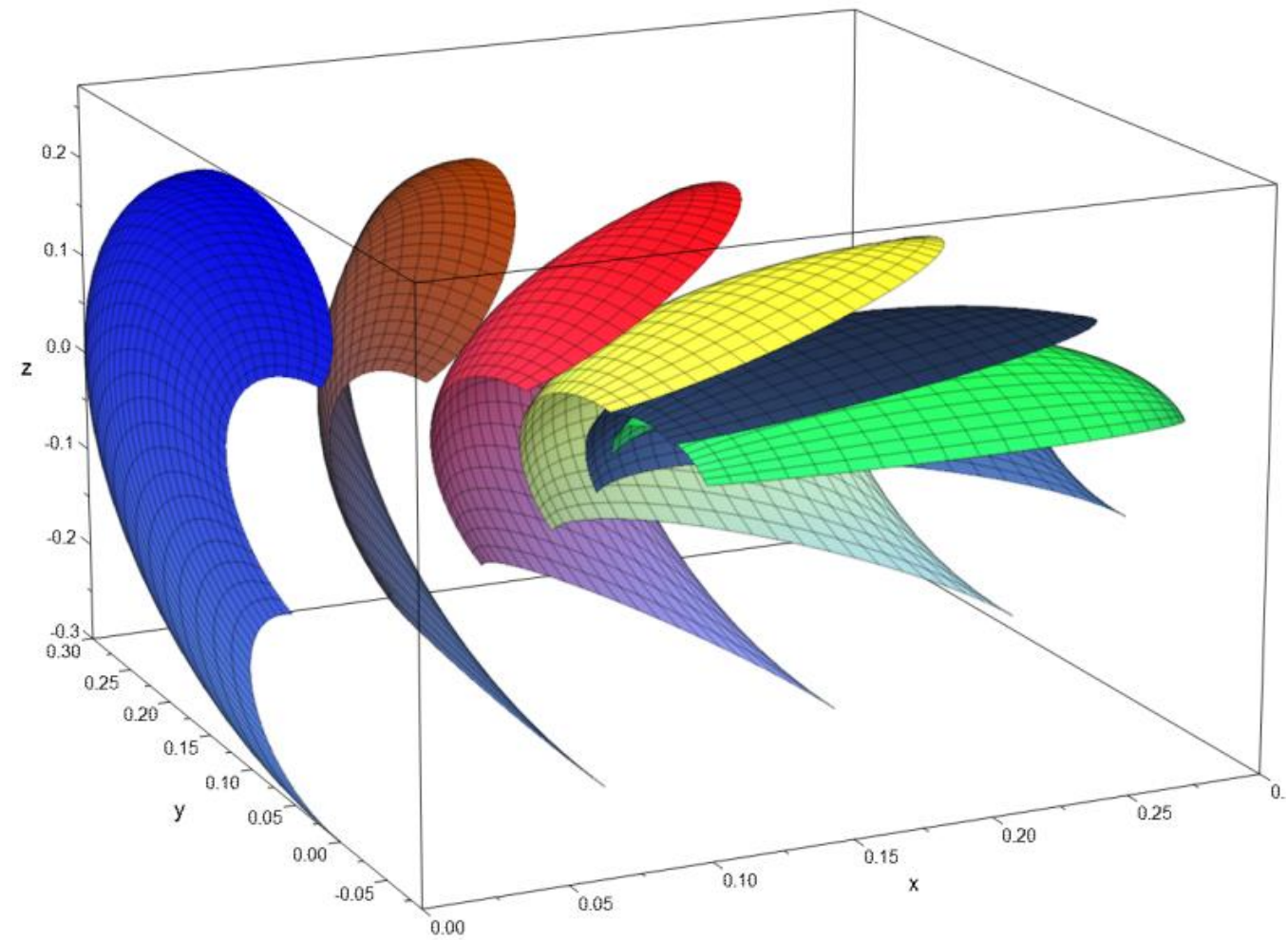

4d) The workspaces of C-manifolds for C-ISA 1 of $\alpha_{1,2}=0$ and C-ISA 2 of $\alpha_{3,4}=\mathrm{i} . \pi / 10$.

Dark Blue: 1, Brown: 2, Red: 3, Yellow: 4, Light Blue: 5, Green: 6

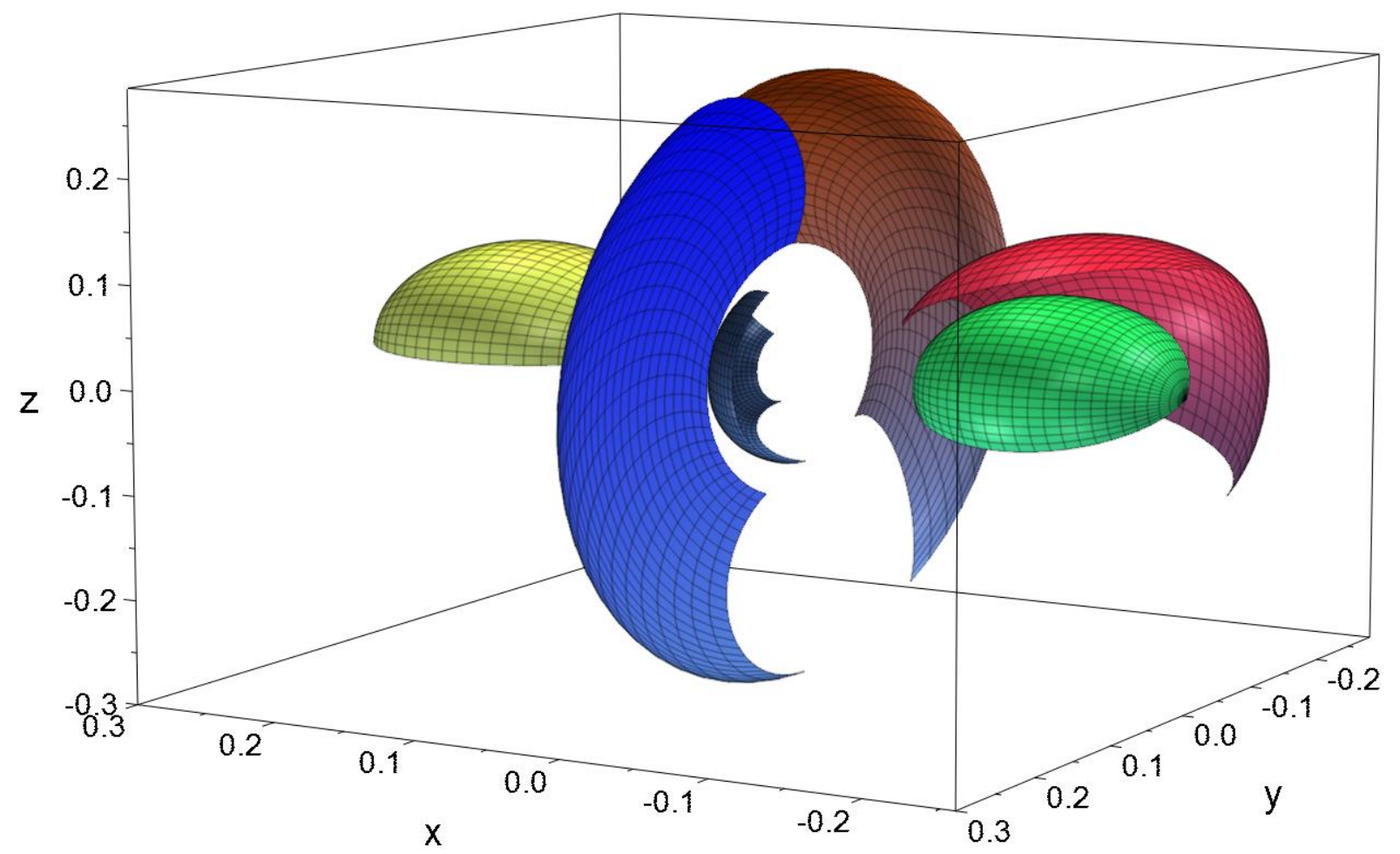

4e) The workspaces of C-manifolds for C-ISA 1 of $\alpha_{1,2}=0$ and C-ISA 2 of $\alpha_{3}=\mathrm{i} . \pi / 10, \alpha_{4}=10 \pi /(\mathrm{i}+1)$ 
Dark Blue: 1, Brown: 2, Red: 3, Yellow: 4, Light Blue: 5, Green: 6

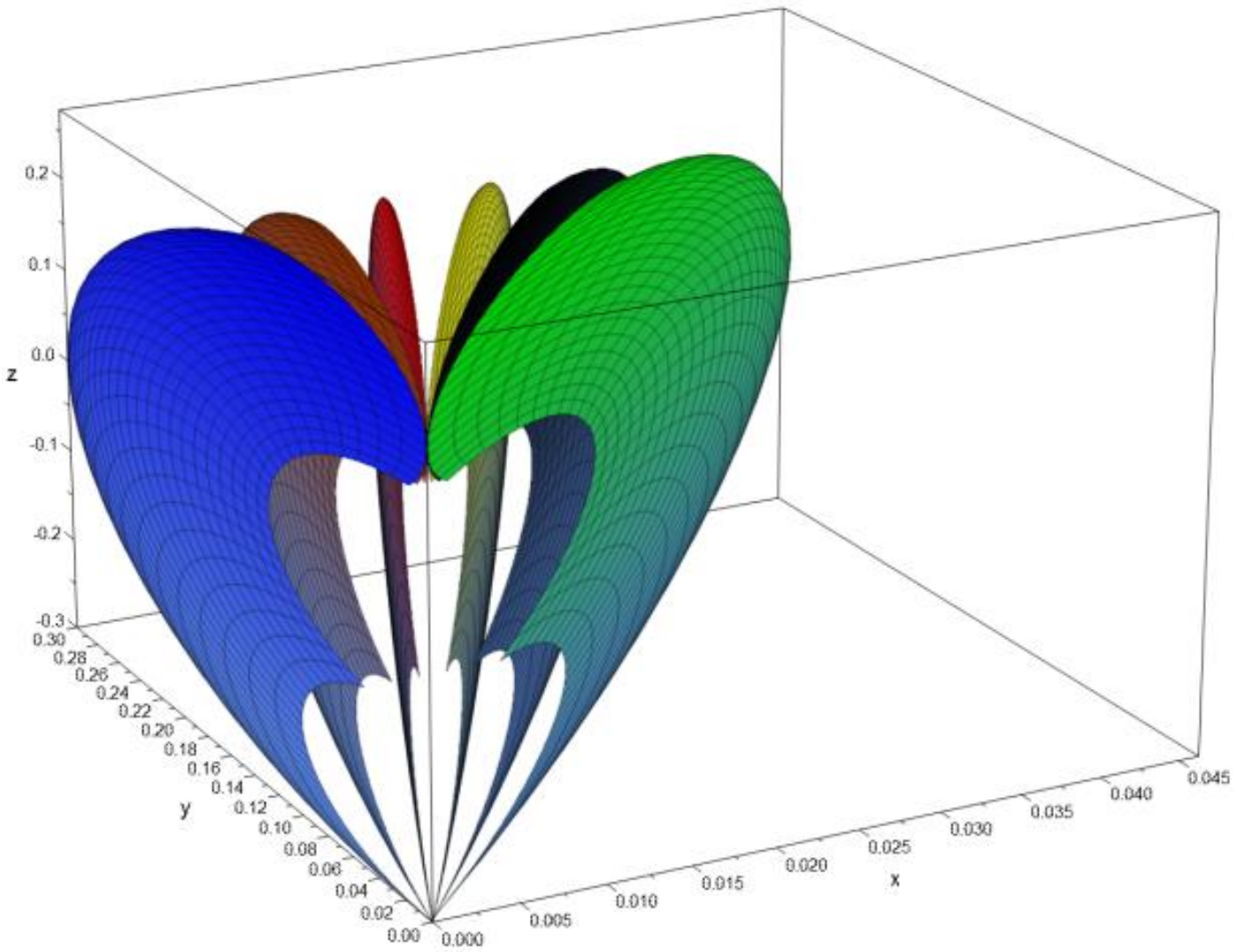

4f) The workspaces of C-manifolds for C-ISA 1 of $\alpha_{1,3}=i . \pi / 100$ and C-ISA 2 of $\alpha_{2,4}=0$.

Dark Blue: 1, Brown: 2, Red: 3, Yellow: 4, Light Blue: 5, Green: 6

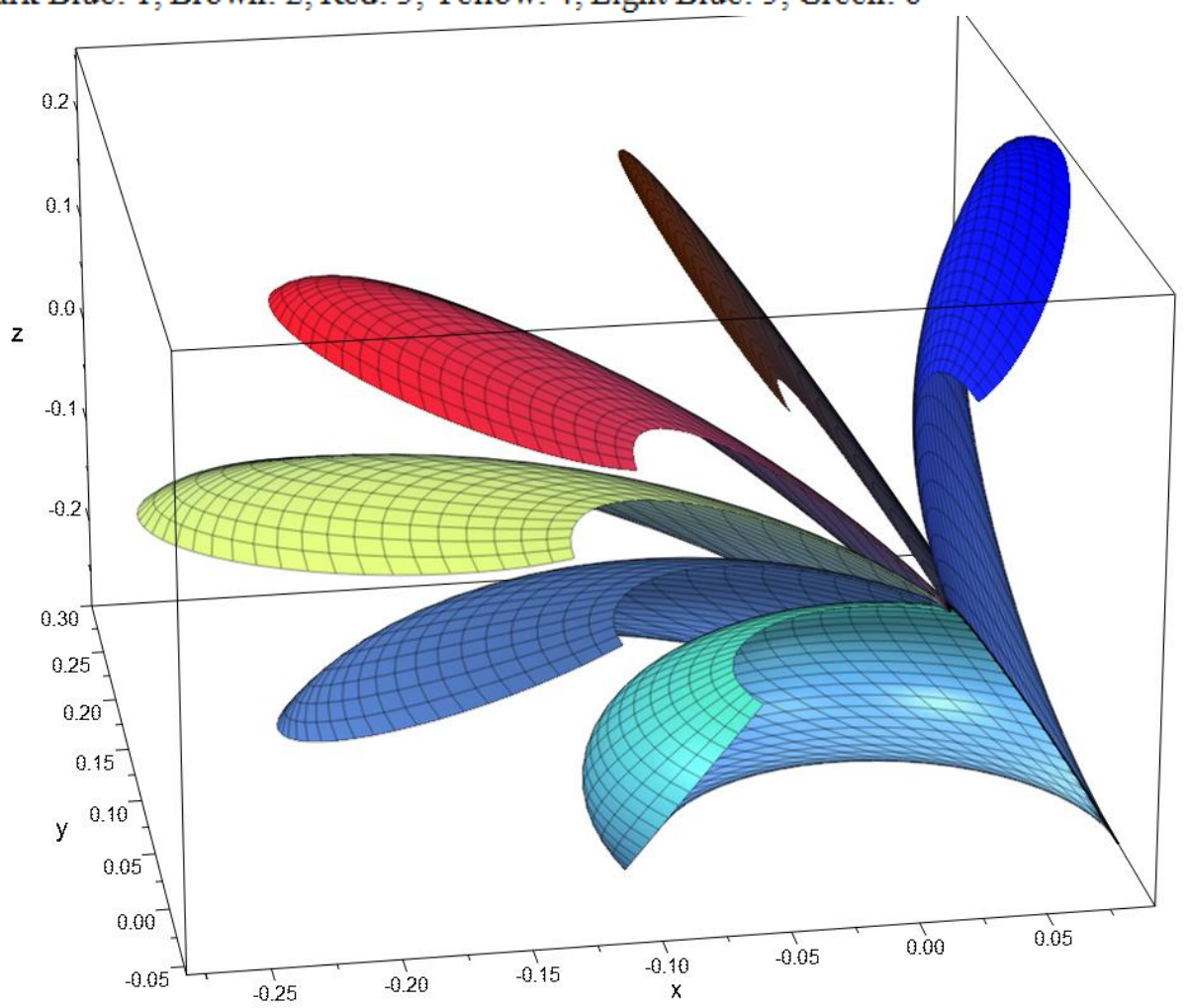

4g) The workspaces of C-manifolds for C-ISA 1 of $\alpha_{1,3}=0$ and C-ISA 2 of $\alpha_{2,4}=\mathrm{i} . \pi / 10$. 
Dark Blue: 1, Brown: 2, Red: 3, Yellow: 4, Light Blue: 5, Green: 6

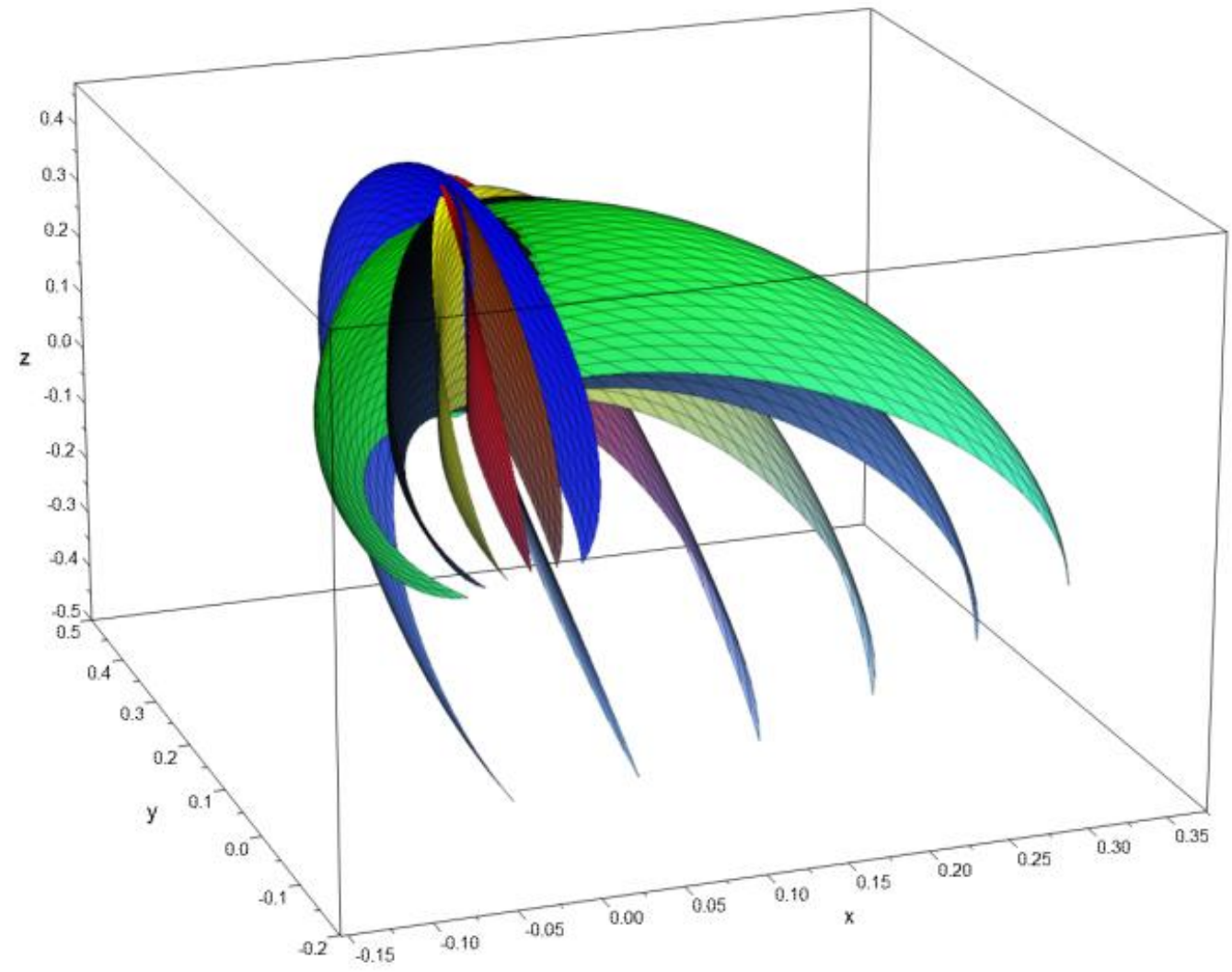

4h) The workspaces of C-manifolds for C-ISA 1 of $\alpha_{1,2}=\mathrm{i} . \pi / 30$ and C-ISA 2 of $\alpha_{3,4}=\mathrm{i} . \pi / 20$.

Dark Blue: 1, Brown: 2, Red: 3, Yellow: 4, Light Blue: 5, Green: 6

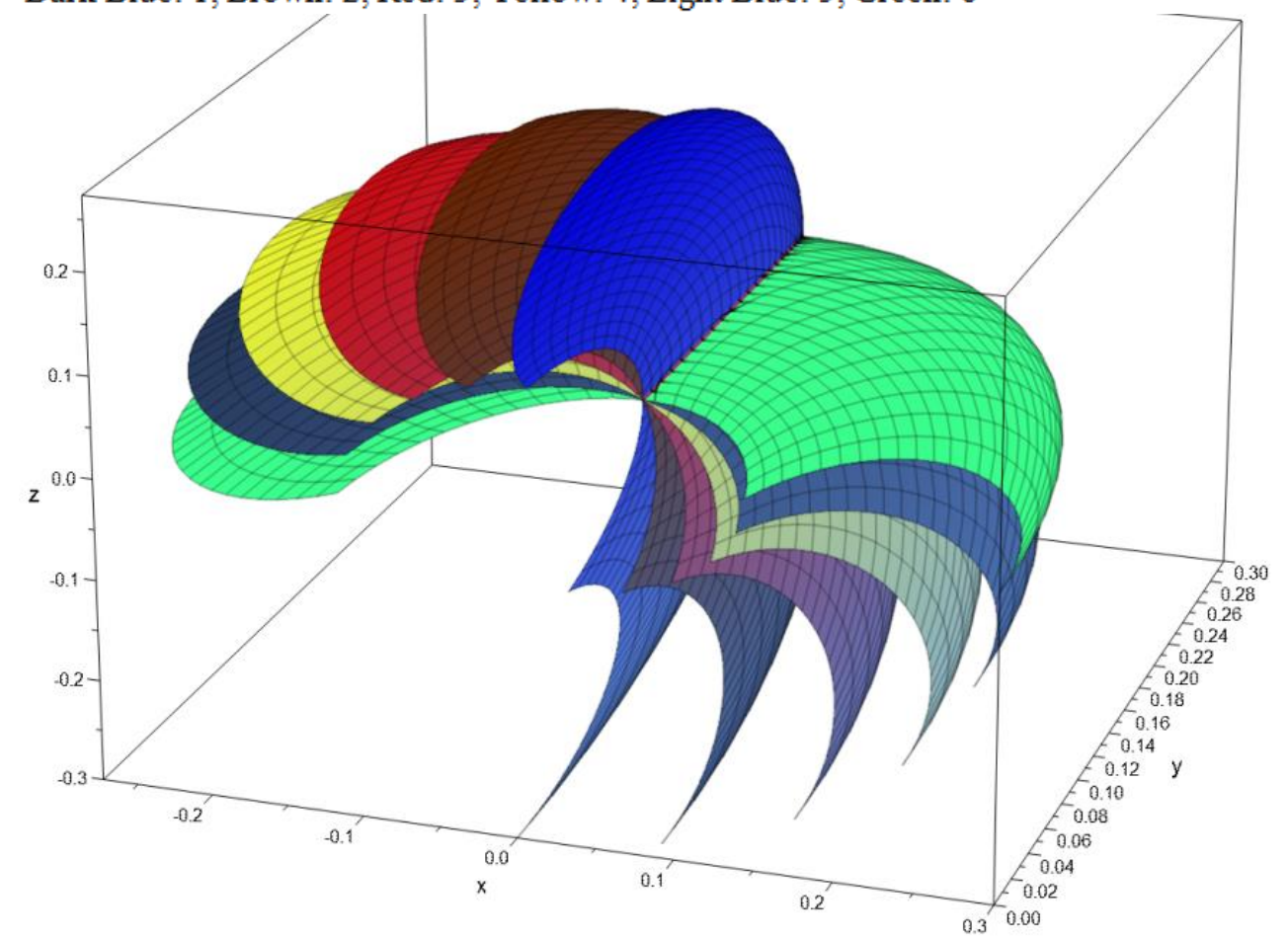

4i) The workspaces of C-manifolds for C-ISA 1 of $\alpha_{1,3}=0$ and C-ISA 2 of $\alpha_{2,4}=i . \pi / 10$. 


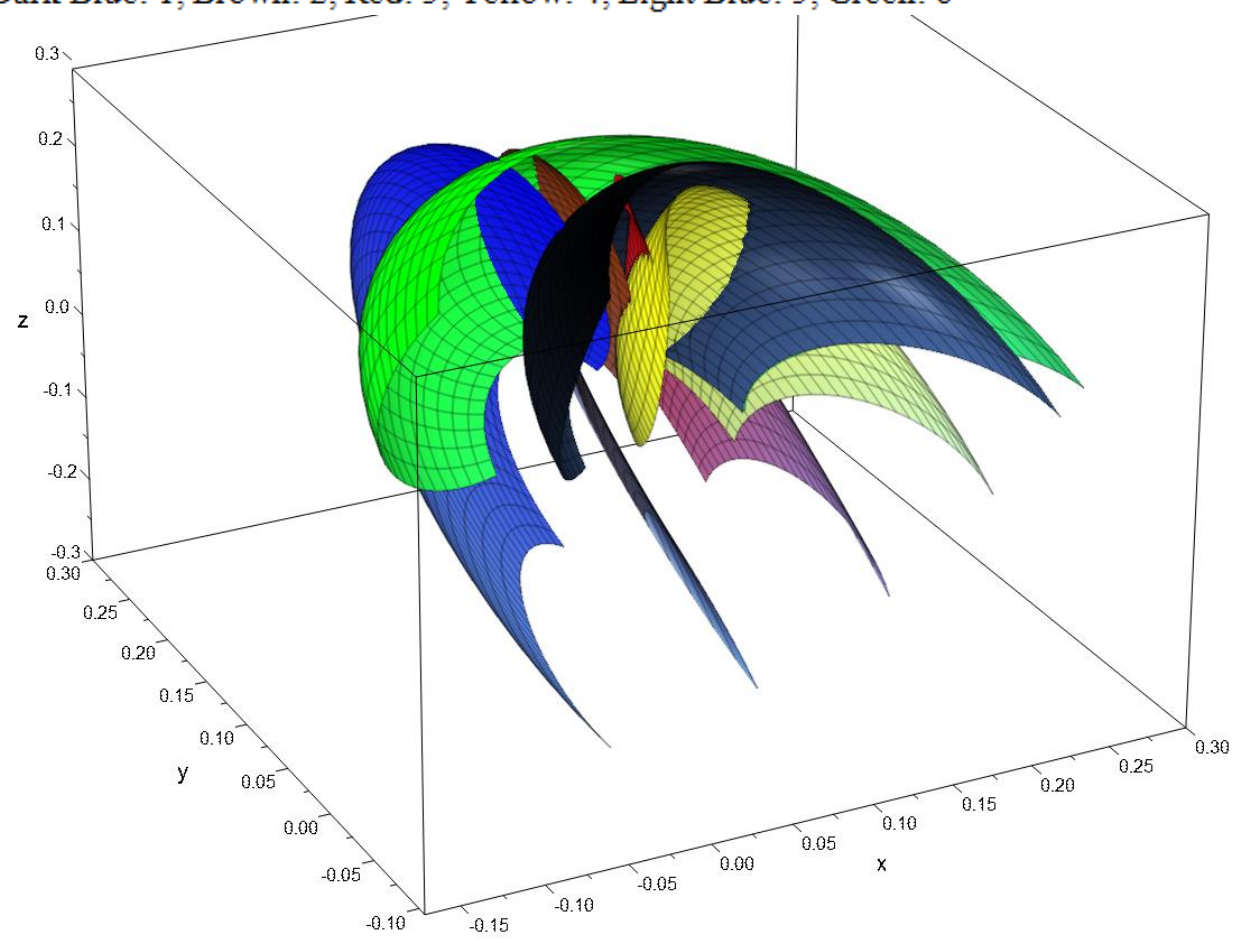

4k) The workspaces of $C$-manifolds for C-ISA 1 of $\alpha_{1,2}=\mathrm{i} . \pi / 10$ and C-ISA 2 of $\alpha_{3,4}=\mathrm{i} . \pi / 10$.

Figure 4. The reduced shape variable values are $\theta_{1}$ and $\theta_{2}=5 \pi / 6$ radian with $i=1-6$ for the workspaces defined as $A d_{\left(g_{\left.1 j_{2}\right)}\right)} \xi_{1 j_{2} 2 j}^{S}$.

If the collaborative functionalities require the concurrent and synchronous motion of the multiple robots for processing the parts in the automation system, the overlapping of the workspace designs contributes to the trajectories for macro modular $C$-manifold accordingly, as indicated in the Figures of the $\mathbf{4} \mathbf{f}-\mathbf{4} \mathbf{k}$. The combination of the orthogonal and parallel axes of rotations for workspace shapes the spatial workspaces of the $3 \mathrm{a}$ and $3 \mathrm{e}$ with no overlapping. The $4 \mathbf{f}$ and $4 \mathbf{g}$ are overlapping on the edge or corner of the workspaces. The $C$-manifolds specified the C-ISA of rule-based $\alpha_{1-4}$ values using differential geometry.

The workspaces in Figure $4 \mathrm{c}$ can depict that parallel 2-RR modular robots established with configurations. They can collaborate to manipulate multiple objects in automation for the planned task spaces. Collaborative robots can be designed using the positions of Figure $4 \mathrm{~b}$ and $4 \mathrm{~d}$ on the same axis for the concentric axis of the workspaces. The parallel workspaces of a flat surface without overlapping of the 2-RR parallel modular robots depict that they can access the same space with the same positional orientation and position parallelly, as shown in Figure 4c. Collaborative robots can exploit designed configurations of Figure $4 \mathrm{~b}$ and $4 \mathrm{~d}$ on the same axis of rotation for workspaces. The configuration-dependent modular design of C-ISA 1 and 2 achieved no overlapping workspaces, parallel workspaces, and concentric axes of workspaces for the six 2-RR modular robots. Thus, the kinematic synchronized motion control of the 2-RR modular robots of macro modular C-manifold accomplish the qualified workspace designs developed, as shown in Figure 3. 
These algorithms describe applying the parametric variables of the $\alpha_{1-4}$, as indicated in the Figures of the 4a- k. The collaborative multi-robots can be integrated based on the workspace results of Figure 4 . Furthermore, the rule-based workspace for task space generates using the kinematics equations developed by the SMILAT method (Sahin, 2021).

\section{Discussions}

The results hereby are classifying the created workspaces via algorithmic workspace programming of the collaborative multi-robots. These classifications herein are the non-overlapping workspaces (a-e), overlapping on the edge of the workspaces (f - g ), overlapping in the middle of the workspaces (h k).

The mathematical rules are designed by the relative positions between $\alpha_{1,2}$ and $\alpha_{3,4}$, between $\alpha_{1,3}$ and $\alpha_{2,4}$. Similarly, the relative positions are arranged among $\alpha_{1,2}, \alpha_{3}$, and $\alpha_{4}$ shape variables. The specified characteristics of the overlappings are explained in the results section as below.

1. The regional overlapping are collaborating on the common diagonal axis wherein the collaboration occurs in diagonal of the six multi-robots

2. The regional overlapping are collaborating on the common axis parallel to y for the six robots.

3. Multiple regional overlappings occurs for the six collaborative 2-RR robots.

4. Overlapping the one edge is collaborating on the y axis where the vertical motion occurs for the robots.

5. Overlappings are collaborating on the two edges of the z-axis.

Rules of the C-ISA 1 and 2 are for C-manifolds as in the Equation of the $A d_{\left(g_{1 j_{-} 1}\right)} \xi_{0_{-} 1 j}^{S}$.

A former study of the author generated the toolbox for the 2-RR modular robot workspace. Previously, the angles were constant in between the twists (Sahin, 2021). The study, herein, aims at the algorithmic workspace programming without considering the angle between C-ISA 1 and 2. This reduction in the level of the constraints can unveil the extended boundaries of the algorithms developable for collaborative multi-robots. The collaboration regions of workspaces herein are definable with the intersecting areas for collaborative trajectories among the six multi-robots. The results indicate the mentioned regions with the rules.

The algorithm design of the workspaces of the modular C-manifold mechanisms of collaborative multi-robot defines the following steps as tasks.

Step 1: Select the sequence of the reduced shape variables for 2-RR collaborative robots.

Step 2: Select the range of the reduced shape variables.

Step 3: Select the collaboration-related proximities between the workspaces for the defined task spaces of the multi-robots.

Step 4: Select one workspace of the C-manifolds with vertical, lateral, diagonal, spatial workspace sequence for the collaborative multi-robot systems of the task spaces. 
Step 5: One can select a combination of the C-manifold surfaces dependent on the task spaces.

Step 6: Define the trajectories of the line paths using the workspaces.

The workspaces design the surfaces with the parameters of the $\left(\alpha_{1}, \alpha_{2}, \theta_{1}\right), i=1-8$, and $\alpha_{1}=\pi / i, \alpha_{2}=0$ $\pi /$ i. The animations describe these $C$-manifold surfaces of $\theta_{1}=0-\pi / i$ while defining the third dimension as an iterative change in perpendicular dimension. The multiple surface animations can be created from the workspaces using three reduced shape variables of $\theta_{1}, \alpha_{1}$, and $\alpha_{2}, \theta_{2}, \alpha_{3}$, and $\alpha_{4}$. The loaded paths are for the generated workspaces of C-manifolds for C-ISA 1 of $\alpha_{1,2}=\mathrm{i} . \pi / 30$ and C-ISA 2 of $\alpha_{3,4}=\mathrm{i} . \pi / 20$.

The depository of the trajectories generates toward the saved workspaces. The workspaces are stored to realize the appropriate paths out of them. The parametric values to form them extracted the algorithms of the saved workspaces for the collaborative robots. The generated workspace of $3 \mathrm{f}$ has the parameters of $\alpha_{1,2}=\mathrm{i} . \pi / 30$ and $\alpha_{3,4}=\mathrm{i} . \pi / 20$. The reduced shape variable values are $\theta_{1}$ and $\theta_{2}=5 \pi / 6$ radian with i $=1-6$ for the workspaces defined as $A d_{\left(g_{1 j_{2} 2}\right)} \xi_{1 j_{-} 2 j}^{S}$.

The algorithm for trajectory 1 obtains using workspace $3 \mathrm{f}$. When $\mathrm{i}=1$ is substituted then the parameter values are $\alpha_{1,2}=1 . \pi / 30$ and $\alpha_{3,4}=1 . \pi / 20$. The lines to follow are including $\theta_{1}=0$ and $\theta_{2}=0$ $5 \pi / 6$ as the parametric algorithm from the workspaces. Additionally, the line generation obtained with the parameter values of the $\theta_{1}=5 \pi / 6$ and $\theta_{2}=0-5 \pi / 6$.

The workspace algorithms are for the collaborative multi-robot trajectory and workspace design with collision avoidance. The algorithm for trajectory six is creatable using workspace $3 \mathrm{f}$. When is $\mathrm{i}=6$, then the parameters have the values of the $\alpha_{1,2}=6 . \pi / 30$ and $\alpha_{3,4}=6 . \pi / 20$.

The trajectories are generatable via $\theta_{1}=0$ and $\theta_{2}=0-5 \pi / 6$. Another path is generated via the values of the $\theta_{1}=5 \pi / 6$ and $\theta_{2}=0-5 \pi / 6$. The boundaries of the workspaces are realized as a group of the created trajectories out of the workspace surfaces.

Workspace algorithms activate the collaborative multi-robot trajectory and workspace design with collision avoidance. The multi collaborative robots actuate using algorithm 1.

Algorithm 1 is defined to shape the trajectories using the created workspaces. The workspaces of $C$ manifolds are for C-ISA 1 of $\alpha_{1,2}=\mathrm{i} . \pi / 10$ and C-ISA 2 of $\alpha_{3,4}=\mathrm{i} . \pi / 10$. The reduced shape variable values are $\theta_{1}=0-5 \pi / 6$ and $\theta_{2}=0-5 \pi / 6$ radians with $i=1-6$ for the workspaces defined as $A_{\left(g_{\left.1 j_{2}\right)}\right)} \xi_{1 j_{-} 2 j}^{S}$.

\section{Conclusion}

This study generates the rules and algorithms for the design of the 2-RR workspaces of the collaborative multi-robots. These mathematical and kinematic rules with the developed algorithm are the novelty of this research article. The results unveil the novel workspaces with the generated algorithms. The multi collaborative robots shall use these algorithms for trajectory generations without collisions. The transitional regions of the intersections in the workspaces are also the state-of-the-art 
value additions for this article herein. These classifications for the transitional path generations can aid the six multi-robots to operate wherein they access the same generated path to collaborate for the task spaces. The collisions are avoidable for the six 2-RR robots with the algorithms developed for trajectory generation while functioning in the environment of the multi robotic systems. The results convince the further investigation of the multi collaborative robot workspace analyses using the developed algorithms herein.

The depository of the trajectories is creatable via the algorithms of the workspaces defined herein, which are expandable into further robotic configurations. The boundaries of the algorithm for the path generations are realized herein for the six collaborative multi-robots. These boundaries determine via the interaction with each other according to the standards.

\section{Acknowledgments}

This research did not receive any specific grant from funding agencies in the public, commercial, or not-for-profit sectors.

\section{Conflict of Interest}

The author declares that there is no conflict of interest.

\section{Author's Contributions}

The contribution of the author is $100 \%$.

\section{References}

Byner C., Matthias B., Ding H. Dynamic speed and separation monitoring for collaborative robot applications - Concepts and performance. Robotics and Computer Integrated Manufacturing 2019; 58: 239-252. https://doi.org/10.1016/j.rcim.2018.11.002

Clark AB., Rojas N. Design and workspace characterisation of malleable robots. 2020 IEEE International Conference on Robotics and Automation (ICRA), Paris, France, 2020;9021-9027, doi: 10.1109/ICRA40945.2020.9197439

Dilibal S., Sahin H., Danquah JO., Faruk EMO., Choi JW. Additively manufactured custom soft gripper with embedded soft force sensors for an industrial robot. International Journal of Precision Engineering and Manufacturing 2021; 22 (4): 709-718.

Elbanhawi M., Simic M. Sampling-based robot motion planning: A review. IEEE Access 2014; 2: 5677.

Feng Z., Hu G., Sun Y., Soon J. An overview of collaborative robotic manipulation in multi-robot systems. Annual Reviews in Control 2020; 49: 113-127.

Henderson AMT., Prazenica RJ. Trajectory generation for a multibody robotic system using the product of exponentials formulation. American Institute of Aeronautics and Astronautics 2021- 
2016. 4 Jan 2021 Forum. https://doi.org/10.2514/6.2021-2016

International Organization for Standardization, ISO/TS15066:2016 - Robots and Robotic Devices Collaborative Robots, 2016.

Krizmancic M., Arbanas B., Petrovic T., Petric F., Bogdan S. Cooperative aerial-ground multi-robot system for automated construction tasks. in IEEE Robotics and Automation Letters 2020; 5( 2): 798-805.

Lakshmanan AK., Mohan RE., Ramalingam B., Anh VL., Veerajagadeshwar P., Tiwarb K., Ilyasa M. Complete coverage path planning using reinforcement learning for Tetromino based cleaning and maintenance robot. Automation in Construction 2020; 112: 1-11.

Le AV., Nhan NHK., Mohan RE. Evolutionary algorithm-based complete coverage path planning for tetriamond tiling robots. Sensors 2020; 20(2): 1-14.

Martínez O., Campa R. Comparing methods using homogeneous transformation matrices for kinematics modeling of robot manipulators. In: Pucheta M., Cardona A., Preidikman S., Hecker R.(eds) Multibody Mechatronic Systems. MuSMe 2021. Mechanisms and Machine Science, 94. Springer, Cham, 2021. https://doi.org/10.1007/978-3-030-60372-4_13

Marvel AJ., Norcross R. Implementing speed and separation monitoring in collaborative robot workcells. Robotics and Computer-Integrated Manufacturing 2017; 44: 144-155.

Moe S., Pettersen KY., Gravdahl JT. Set-based collision avoidance applications to robotic systems. Mechatronics 2020; 69: 1-19. https://doi.org/10.1016/j.mechatronics.2020.102399

Olesen AS., Gergaly BB., Ryberg EA., Thomsen M.R., Chrysostomou D. A collaborative robot cell for random bin-picking based on deeplearning policies and a multi-gripper switching strategy. Procedia Manufacturing 2020; 51: 3-10. https://doi.org/10.1016/j.promfg.2020.10.002.

Queralta JP., Taipalmaa J., Pullinen BC., Sarker VK., GIA TN., Tenhunen H., Gabbouj M., Westerlund T. Collaborative multi-robot search and rescue: planning, coordination, perception, and active vision. In IEEE Access 2020; 8: 191617-191643.

Rajesh KM., Anandu R., Sakthiprasad KM. Comparison of planned path and travelled path using ros navigation stack. 2020 International Conference for Emerging Technology (INCET) 1-6, 2020.

Sahin $\mathrm{H}$. The modular nonoverlapping grasp workspaces and dynamics for the grippers using the micro and macro C-Manifold design. Journal of Scientific \& Industrial Research 2021; 9: 766-776.

Su H., Liu S., Zheng B., Zhou X., Zheng K. A survey of trajectory distance measures and performance evaluation. The International Journal on Very Large Data Bases 2020; 29: 3-32.

Thalamy P., Piranda B., Bourgeois J. A survey of autonomous self-reconfiguration methods for robotbased programmable matter. Robotics and Autonomous Systems 2019; 120: 1-17.

Wang J., Meng MQH. Optimal path planning using generalized voronoi graph and multiple potential functions. IEEE Transactions on Industrial Electronics 2020; 67(12): 10621-10630.

Wang X., Liu X., Chen L., Hu H. Deep-learning damped least squares method for inverse kinematics of redundant robots. Measurement 2021; 171: 108821, ISSN 0263-2241. 
Xiao F., Li G., Jiang D., Xie Y., Yun J., Liu Y., Huang L., Fang Z. An effective and unified method to derive the inverse kinematics formulas of general six-DOF manipulator with simple geometry. Mechanism and Machine Theory 2021; 159: 1-14.

Yang S., Wen H., Hu Y., Jin D., Coordinated motion control of a dual-arm space robot for assembling modular parts. Acta Astronautica. Acta Astronautica 2020; 177: 627-638. 